\title{
Nucleotide diversity inflation as a genome- wide response to experimental lifespan extension in Drosophila melanogaster
}

\author{
Pawel Michalak ${ }^{1 *}$, Lin Kang ${ }^{1}$, Pernille M. Sarup ${ }^{2}$, Mads F. Schou ${ }^{2}$ and Volker Loeschcke ${ }^{2^{*}}$
}

\begin{abstract}
Background: Evolutionary theory predicts that antagonistically selected alleles, such as those with divergent pleiotropic effects in early and late life, may often reach intermediate population frequencies due to balancing selection, an elusive process when sought out empirically. Alternatively, genetic diversity may increase as a result of positive frequency-dependent selection and genetic purging in bottlenecked populations.

Results: While experimental evolution systems with directional phenotypic selection typically result in at least local heterozygosity loss, we report that selection for increased lifespan in Drosophila melanogaster leads to an extensive genome-wide increase of nucleotide diversity in the selected lines compared to replicate control lines, pronounced in regions with no or low recombination, such as chromosome 4 and centromere neighborhoods. These changes, particularly in coding sequences, are most consistent with the operation of balancing selection and the antagonistic pleiotropy theory of aging and life history traits that tend to be intercorrelated. Genes involved in antioxidant defenses, along with multiple IncRNAs, were among those most affected by balancing selection. Despite the overwhelming genetic diversification and the paucity of selective sweep regions, two genes with functions important for central nervous system and memory, Ptp10D and Ank2, evolved under positive selection in the longevity lines.
\end{abstract}

Conclusions: Overall, the 'evolve-and-resequence' experimental approach proves successful in providing unique insights into the complex evolutionary dynamics of genomic regions responsible for longevity.

\section{Background}

Developing the evolutionary theory of aging has been a key endeavor since the very beginning of the modern evolutionary synthesis $[1,2]$. Two major non-mutually exclusive models of how aging can originate and evolve have been formulated. Both of them rest on the fact that natural selection is weak at old age due to small cohort size and declining contributions to reproduction. One stems from Medawar's ideas [3] that drift and mutation accumulation results in the loss of late-acting beneficial alleles or the emergence of late-acting deleterious alleles $[2,4]$. Another is based on Williams's model of pleiotropy [5] in which aging evolves as a consequence of pleiotropic effects of some genes that are beneficial early in life and

\footnotetext{
* Correspondence: pawel@vbi.vt.edu; volker@bios.au.dk

${ }^{1}$ Biocomplexity Institute, Virginia Tech, 1015 Life Science Circle, Blacksburg, VA 24061, USA

2Department of Bioscience, Aarhus University, Ny Munkegade 114-116, Aarhus DK-8000, Denmark
}

then harmful at later ages. A corollary to the antagonistic pleiotropy theory of aging is that late-life selection operating on genes with pleiotropic effects will lead to the establishment and maintenance of genetic polymorphism, effectively becoming balancing selection [6]. However, the conditions for balancing selection due to antagonistic pleiotropy are fairly restrictive compared with balancing selection due to selective pressures varying in time and space [7-9].

While genomic studies now suggest balancing selection might be relatively common, to date most evidence for it remains indirect. For example, the relative levels of the effects of artificial selection on the population mean and inbreeding depression for the selected trait can separate variation maintained largely by mutation-selection balance and variation from a contribution from alleles at intermediate frequencies (tantamount to balancing selection) [10]. Intermediate allele frequencies played a key role in a selection experiment on female fecundity 
in Drosophila melanogaster [11], similar to selection on several traits in the monkeyflower Mimulus guttatus $[12,13]$. Balancing selection could also account for the genetic variance in viability linked to the small fourth chromosome of $D$. melanogaster [14, 15], and polymorphism in numerous sites across Drosophila genomes seem to be maintained by balancing selection due to seasonal oscillations in climate [16].

Although through a different mechanism, the mutation accumulation theory of aging also predicts that polymorphism will be increased among genes with age-specific effects. As long as late-expressed alleles have no or very little differential effect on fitness, and the older the age the less fitness effects are indeed expected, such alleles will be subject to mostly neutral evolution, notwithstanding their discernible phenotypes [6]. Other processes, such as positive frequency-dependent selection [17] and purging selection against homozygotes for deleterious alleles affects [18] may also boost genetic polymorphism, especially when a reduction in population size is involved.

To test the prediction that selection for longevity increases genetic diversity, here we survey genome-wide patterns of nucleotide polymorphism in Drosophila melanogaster experimentally selected for increased lifespan [19-21]. Unsurprisingly, Drosophila have been a historically important system for investigating the genetic underpinnings of longevity $[22,23]$, and recently have become an even more attractive model owing to their tractable, relatively small genomes. In addition to dissecting the genetic basis of longevity, Drosophila have been indispensable in investigating the physiology of aging [24-26].

Experimental evolution employs well-established selection protocols to enforce phenotypic divergence, which coupled with the genome-wide analysis ('evolve-and-resequence') may narrow down the candidate target regions under selection, and provide a powerful alternative to genomewide association studies (GWASs) and linkage mapping experiments as strategies to link genotype with phenotype [27-32]. A major advantage of experimental evolution compared to other evolutionary approaches is its ability to distinguish between stochastic and deterministic effects based on parallel replicates under controlled conditions. Another advantage of experimental evolution is that selection for life history traits, such as longevity, under a controlled laboratory environment with ample food, reduced competition and other antagonistic interactions is less likely to be affected by the constraints posed by trade-offs in suboptimal environments under natural conditions $[33,34]$.

\section{Results}

\section{Variant discovery analysis}

Three longevity lines after 48 generations of selection and three parallel control lines (standard laboratory conditions without selection) were sequenced using pooled genomes per line (Pool-seq). We found a total of $1,497,961$ polymorphic sites, $1,212,878$ of which were heterozygous in all longevity lines and 1,050,542 were heterozygous in all control lines. A total of 192,558 SNPs were homozygous in all longevity lines while being heterozygous in at least one of the control lines, and, conversely, 305,601 SNPs were fixed in all control lines while heterozygous in at least one of the longevity lines. Only 169 SNPs were fixed in all selection lines with an alternative allele fixed in all control lines. Average SNP-based $\mathrm{F}_{\mathrm{ST}}$ estimates were the lowest between the longevity lines (0.082) and the highest in controllongevity pairwise comparisons (0.124), compared with those between control lines (0.104). The inspection of site frequency spectra (SFSs) indicates that there were substantially more alleles with intermediate frequencies in the longevity lines than control lines (Fig. 1).

\section{Heterozygosity and Tajima's D analysis}

Overall, mean heterozygosity was $21 \%$ higher in longevity lines relative to control lines, across all genomic regions, including coding sequences (Fig. 2a). The highest heterozygosity increase $(\sim 77 \%)$ was recorded for chromosome 4 and the lowest (17\%) for chromosome arm 2R (Fig. 3). The genome-wide profiles of Tajima's $\mathrm{D}$ values produced a similar result, with significant differences (Mann-Whitney test, $P<0.001)$ between control and longevity lines across all genomic regions (except the promoter region, Fig. 4) and across all chromosomes (Fig. 5), implying an extensive increase of nucleotide diversity in the selected lines. The highest Tajima's D values were recorded in CDSs and chromosome $2 \mathrm{~L}$ of the longevity lines (Fig. 5). The difference in heterozygosity and Tajima's D between control and longevity lines was most pronounced in the centromere regions ( $2 \mathrm{Mb}$ each) of chromosomes 2 and 3 (47\% heterozygosity increase in longevity lines compared with the $19 \%$ increase in non-centromere regions), and the $\sim 71$ $\mathrm{Kb}$ yellow-achaete-scute complex near the tip of chromosome X (2,281\% increase compared with $47 \%$ increase along the rest of the chromosome $\mathrm{X}$ ) (Table 1).

We then ranked genes according to the increase in Tajima's D-values in the longevity lines relative to control lines (Table 2). One of the top genes with differential D values was intrepid (intr) encoding a serine protease homolog, involved in negative regulation of female mating receptivity and sperm storage [35]. Other genes with differential $\mathrm{D}>2$ included PH4alphaNE3 and CG31021, both involved in the oxidation-reduction process, Prosbeta5R1having endopeptidase activity, and tailless $(t l l)$, a transcription factor playing a role in a variety of developmental and metabolic processes. Overall, oxidoreductase and dioxygenase activities were the most significant GO term enrichments $(P<0.01)$ among genes with Tajima's $D$ values increased in the 

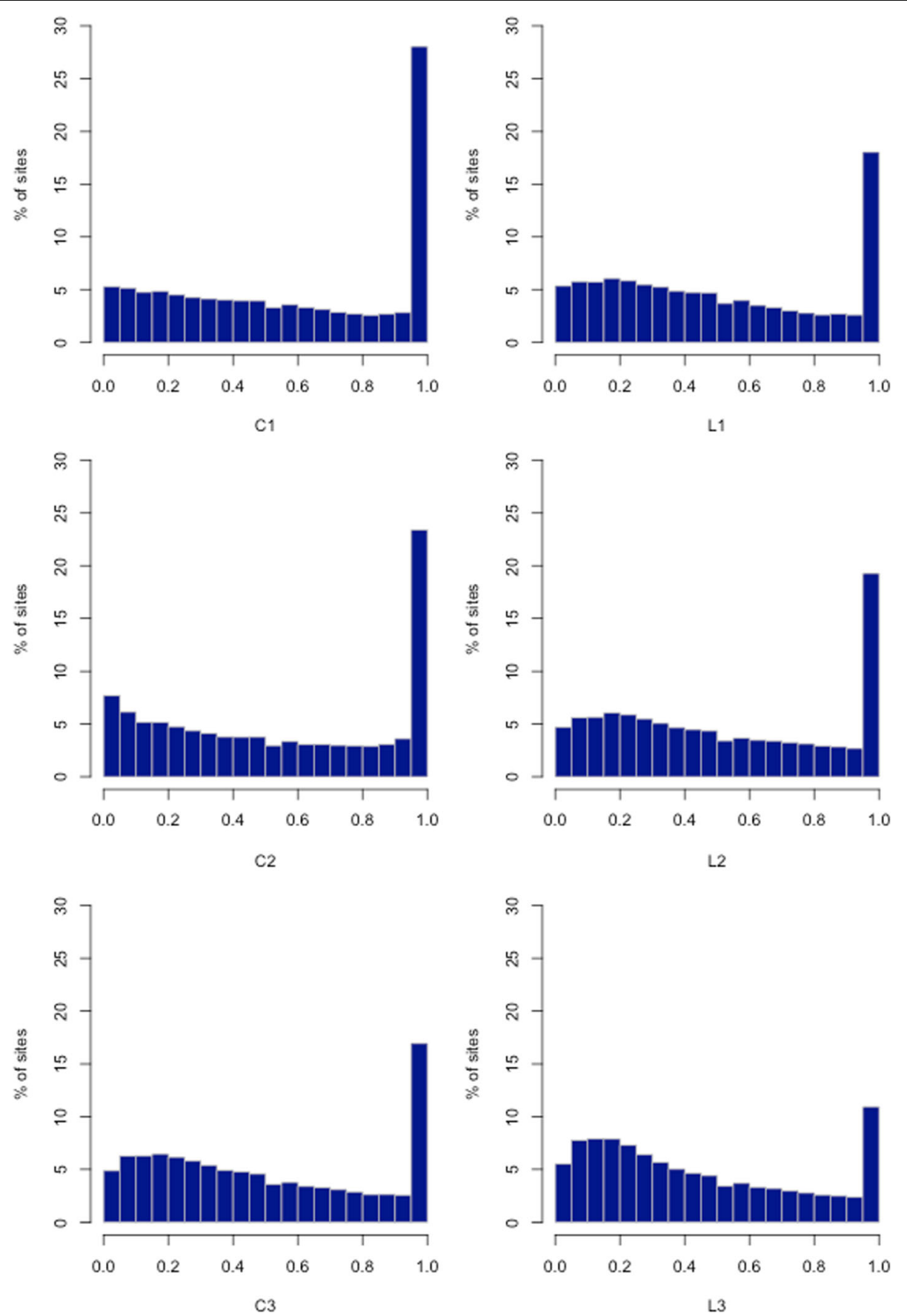

Fig. 1 Site frequency spectra (SFSs) in three control (C1-C3) and three longevity (L1-L3) Drosophila melanogaster lines

longevity lines (Additional file 1: Table S1). Among the top 200 genes with the largest difference in Tajima's D, as many as $32(16 \%, P=0.058$, Additional file 1: Table S1) were long non-coding RNAs (lncRNAs). In addition, a similar pattern of differentiation was found in eight small nucleolar RNAs and three miRNAs (mir-961, mir-968, and mir-2501, $P=0.018$, Additional file 1 : Table S1).
Simulations of neutral evolution and positive frequencydependent selection

Since the longevity lines had an increasingly longer lifespan and the control lines thus had been through 94 more generations at the same time, it was necessary to control for the difference and determine a baseline (95\% CIs) for heterozygosity and Tajima's D changes in control lines due to drift effects alone. Simulations of 


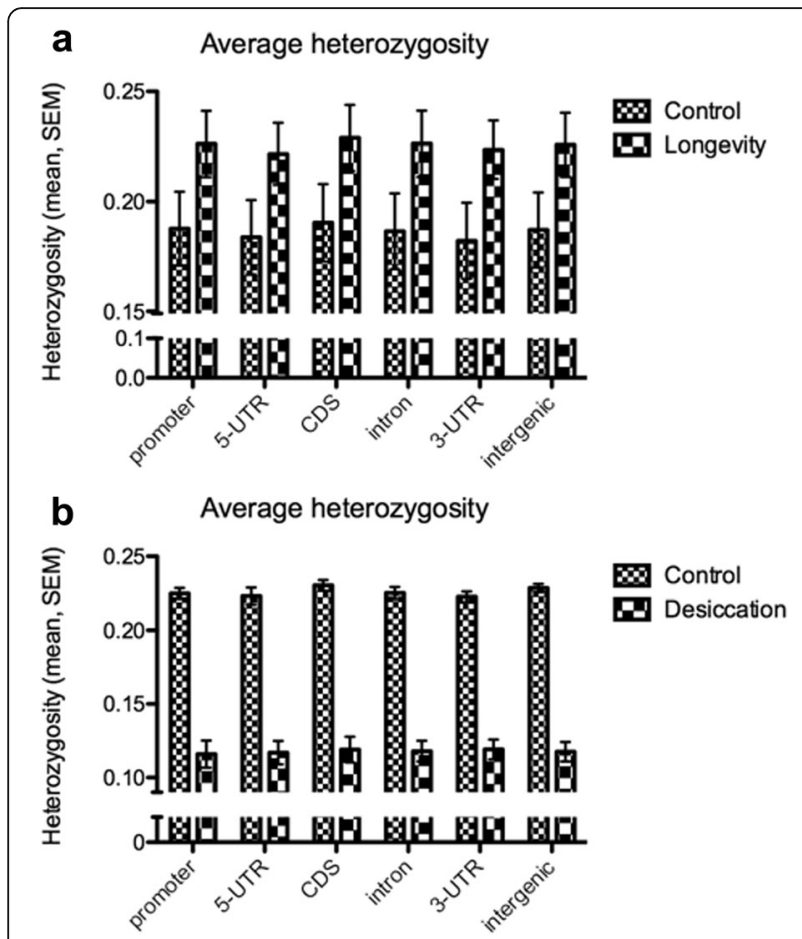

Fig. 2 Average heterozygosity ( $(\mathrm{SEM})$ across various genomic regions in (a) control and longevity D. melanogaster lines, compared with (b) an experimental evolution system selected for desiccation resistance [42]

neutral evolution under conditions mimicking our experimental system, based on genomic variation within the DGRP2, showed on average a $9.88 \% \pm 0.02 \%$ (95\% CIs) heterozygosity decrease in the group that went through $\sim 2 \mathrm{x}$ more (201) generations than the other group (107) (Additional file 2: Figure S1). Drift effects on Tajima's D after the 94 additional generations were negligible: $-0.08 \% \pm 0.61 \%$ (95\% CIs) change. Separately, we also tested effects of positive frequency-dependent selection in conjunction with a bottleneck, which might enhance linkage disequilibrium (LD) and create selection for the commoner allele at the selected locus, potentially leading to an increase of heterozygosity in

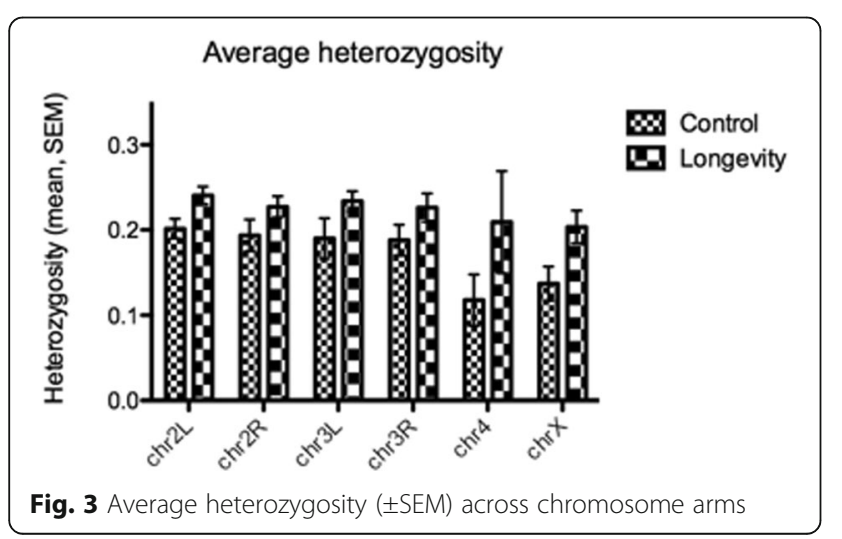

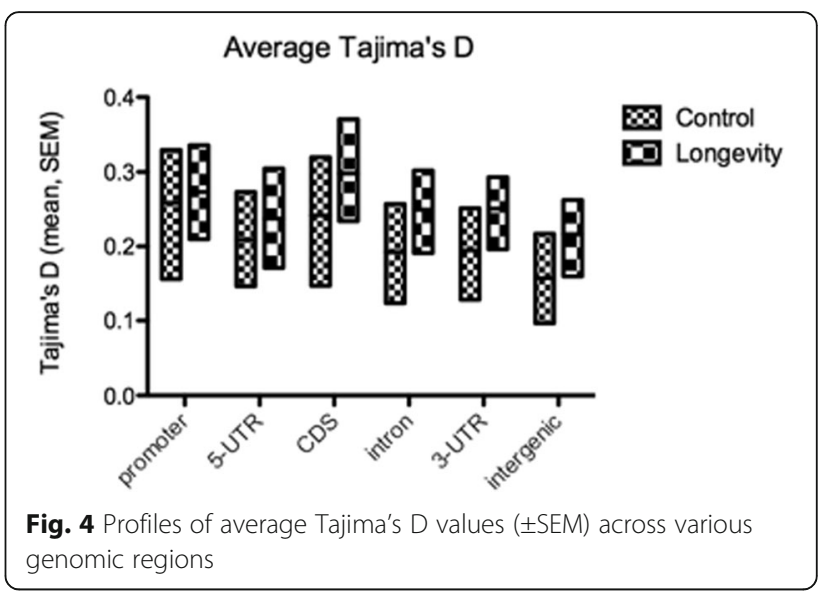

linked neutral loci. However, no increase of heterozygosity due to a stronger selection pressure was observed, compared with low/no selection under various recombination rates (Additional file 3: Figure S2).

\section{Signatures of positive selection}

Genomic regions of depleted genetic variation corresponding to putative selective sweeps were highly reduced in the selected lines (Fig. 6). One protein receptor gene (Ptp10D) belonging to the protein-tyrosine phosphatase family was under significant positive selection in the comparison between selection and control groups $(\mathrm{Ka} / \mathrm{Ks}=9$, McDonaldKreitman test $p=0.0217)$. Ptp10D is responsible for central nervous system development [36], axon guidance [37], and long-term memory [38]. Another gene with $\mathrm{Ka} / \mathrm{Ks}=4$ (but MKT $p>0.05$ ) was Ank2, involved in axon extension [39], neuron cellular homeostasis [39], sensory perception of pain [40] and short-term memory [41].

\section{Discussion}

Strikingly, genome differentiation patterns produced by selection for increased lifespan are opposite to those we observed in selection for desiccation tolerance (Fig. 2b), evolving under strong positive selection [42]. Selection for longevity led to $>20 \%$ genome-wide increase in

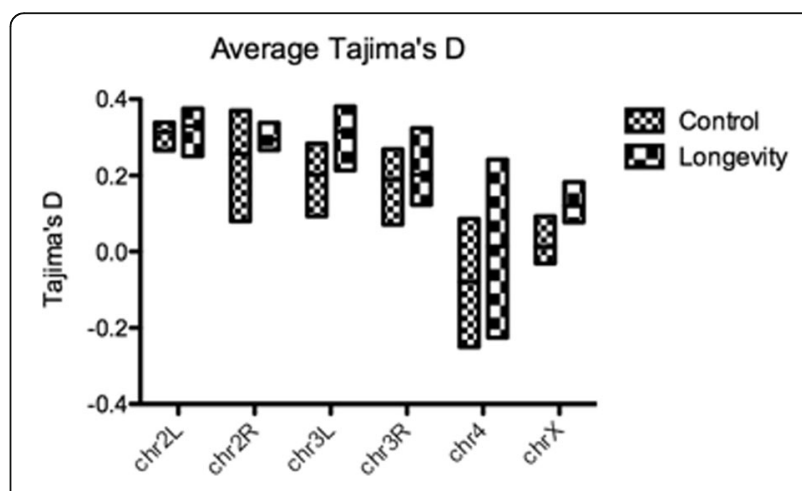

Fig. 5 Profiles of average Tajima's D values ( \pm SEM) across chromosomes 
Table 1 Mean heterozygosity and Tajima's D estimates in regions expected to differ in the levels of recombination, including $2 \mathrm{Mb}$ neighborhoods of autosome centromeres and the $\sim 71 \mathrm{~Kb}$ yellow-achaete-scute complex (Y-AS-C) on chromosome $X$

\begin{tabular}{lllll}
\hline Heterozygosity & & & & \\
\hline & Centromeres & Non-centromeres & Y-AS-C & Non-Y-AS-C \\
\hline Longevity lines & 0.244 & 0.231 & 0.381 & 0.203 \\
Control lines & 0.166 & 0.194 & 0.016 & 0.138 \\
Tajima's D & & & & \\
Longevity lines & -0.053 & 0.292 & 0.214 & 0.115 \\
Control lines & -0.163 & 0.251 & -0.201 & 0.011 \\
\hline
\end{tabular}

heterozygosity relative to control lines, with coding sequences being proportionally more affected than other genomic regions. These results are inconsistent with neutral evolution, given the parallelism in responses across replicates within each group, and the experimental design controlling for numbers of flies used in each generation. Even if effective population sizes $\left(\mathrm{N}_{\mathrm{e}}\right)$ deviated from the experimental numbers of flies, $\mathrm{N}_{\mathrm{e}}$ was presumably lower

Table 2 List of top differentiated genes ranked according to the increase in Tajima's D-values in the longevity lines relative to control lines

\begin{tabular}{llcl}
\hline Gene Symbol & Diff_D & Selection_D & Control_D \\
\hline CG42591 & 2.586 & 1.4701 & -1.1159 \\
SnoRNA:Psi28S-3378 & 2.5005 & 1.5192 & -0.9813 \\
CR43358 & 2.4878 & 1.6261 & -0.8617 \\
CG43880 & 2.4578 & 1.1891 & -1.2687 \\
intr & 2.4081 & 1.914 & -0.4941 \\
CG9168 & 2.3787 & 1.3942 & -0.9845 \\
Or94a & 2.3183 & 1.9587 & -0.3597 \\
CR44225 & 2.305 & 1.3609 & -0.9441 \\
CR45322 & 2.3025 & 1.3841 & -0.9184 \\
CG34432 & 2.2633 & 1.6055 & -0.6578 \\
PH4alphaNE3 & 2.2457 & 1.594 & -0.6518 \\
CR44236 & 2.2203 & 1.2507 & -0.9696 \\
CG4763 & 2.1983 & 1.6423 & -0.556 \\
Prosbeta5R1 & 2.1706 & 1.5612 & -0.6095 \\
CG31021 & 2.137 & 1.52 & -0.617 \\
CG31093 & 2.1198 & 1.452 & -0.6678 \\
snoRNA:Psi18S-1389b & 2.0825 & 1.2012 & -0.8812 \\
CG15398 & 2.0724 & 1.5682 & -0.5042 \\
tIl & 2.0553 & 1.0246 & -1.0307 \\
CR44713 & 2.0283 & 0.7839 & -1.2444 \\
CR44714 & 2.0283 & 0.7839 & -1.2444 \\
Or94b & 2.0223 & 1.4939 & -0.5284 \\
CG32320 & 2.0082 & 0.818 & -1.1902 \\
\hline & & & \\
\hline
\end{tabular}

in selected lines, as some of the living old flies were likely past reproduction. Increased variance in reproductive output compared to young flies would lower $\mathrm{N}_{\mathrm{e}}$ even further, which is in contrast to our observations. However, control lines went through approximately twice as many generations as selection lines at the same time, as a consequence of increasingly extended generation time in the selected group. Our simulations show that although this difference in generation numbers and associated drift effects accounted for approximately $50 \%$ of the observed decline in heterozygosity estimates for control lines, Tajima's D estimates were essentially unaffected. Mutation accumulation effects alone are also unlikely to explain the pattern of variation, due to the moderate population sizes and generation numbers used in this experimental evolution system.

Balancing selection, which entails heterozygote advantage, negative frequency dependence or spatiotemporal heterogeneity (or a combination of any of these), thus emerges as the most likely mechanism capable of producing the increase in genetic diversity. By providing a means to maintain genetic variation, balancing selection is especially relevant to diversification of phenotypic variation in natural populations, including variation in life history traits, such as longevity. However, despite longstanding interest in the process of balancing selection, its prevalence, the extent of footprints in the genome, and evolutionary significance remain largely unknown due to challenges related to its detection [43-46]. Balancing selection is expected to boost neutral polymorphism in linked genome regions, in inverse proportion to their genetic map distances from the selected site, but the size and distribution of genomic regions showing indirect footprints of balancing selection have been unknown [45]. Our results prove that such effects at the genome-wide level can be pervasive and rapid, invoking newly established neutral polymorphisms from standing genetic variation.

Antagonistic pleiotropy theory of aging involves selection operating on genes with pleiotropic effects that can be subject to balancing selection [6], and some agingrelated genes in Drosophila, such as $D d c$, were in fact implicated in the evolution under balancing selection [47]. Life history traits in general evolve as a result of intricate trade-offs, or negative correlations, and antagonistic pleiotropy has been believed to play a central role in this process $[6,48,49]$. At the phenotypic level, there are two critical sources of correlations characterizing aging: the negative correlation between lifespan and reproduction [50-52], and the positive correlation between lifespan and stress tolerance $[23,53]$. Antagonistic and non-antagonistic mechanisms of balancing selection are predicted to differentially affect population genomic signatures of recent balancing selection, with weaker 


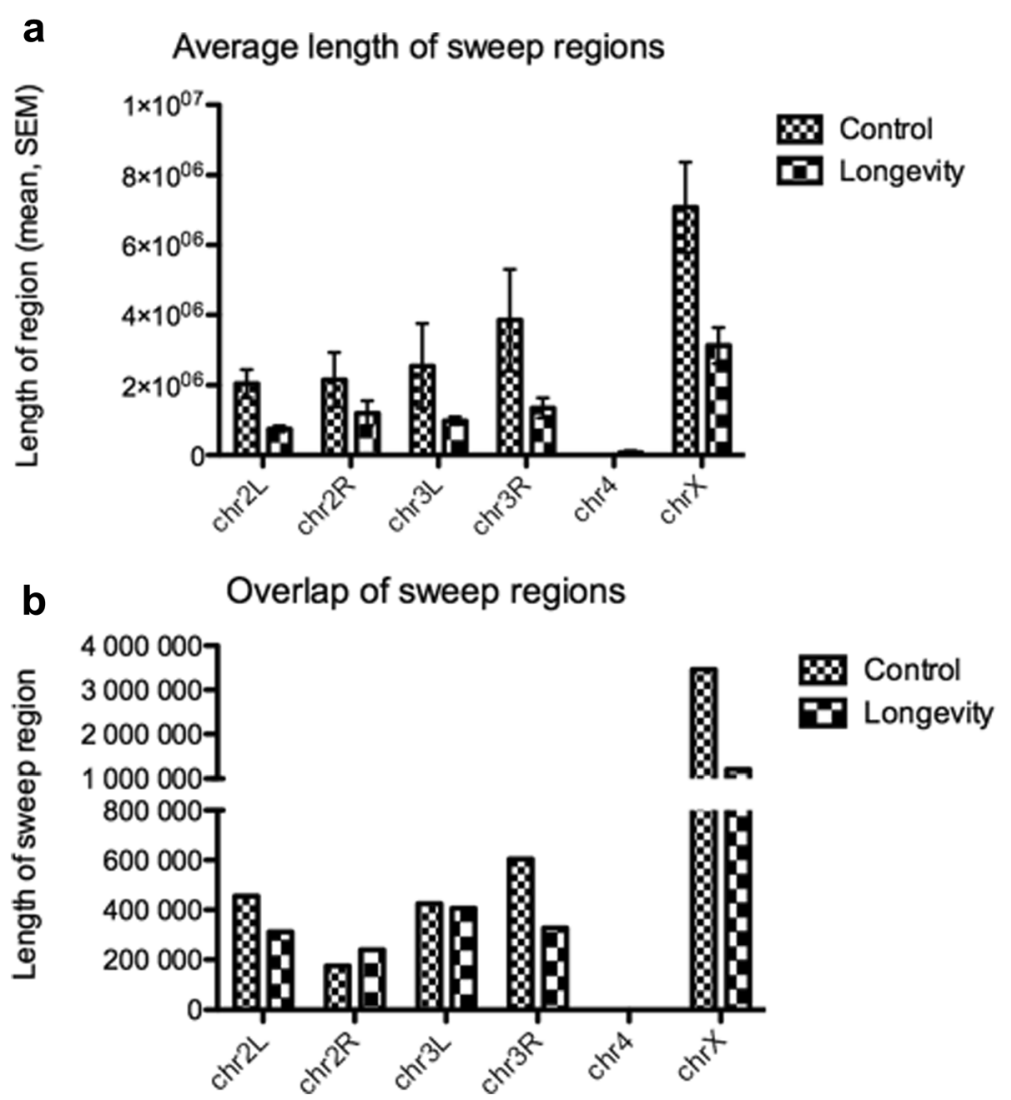

Fig. 6 Sweeps found in the Longevity group and the Control group. Average length ( \pm SEM) of sweep regions (a) and length of shared sweep regions (b) for Longevity and Control groups by chromosomes

signatures under antagonism [9]. Non-antagonistic processes entail partial selective sweeps that proceed rapidly, which results in stronger hitchhiking effects relative to partial sweeps from antagonistic selection [9]. The longevity lines exhibit very weak sweep signatures, even compared with the control lines, a pattern consistent with the antagonistic evolution.

However, even though antagonistic pleiotropy appears as a likely source of balancing selection accounting for the observed pattern of polymorphisms, the set of conditions under which this mechanism can be the sole driver of balancing selection without any temporal change in selection are limited [7-9]. Many insect species go through multiple generations per year, whereby different generations are exposed to different seasonal selection landscapes $[16,54]$, which is likely to result in a difference in optimal age of reproduction between cohorts. This change in selection landscape was mimicked in this study, as selection for longevity was relaxed every other generation, resulting in a substantial increase in longevity without a decrease in the reproductive capacity in early life [20]. We therefore argue that temporal shifts in selection coefficients on genes showing antagonistic pleiotropy for early life and late life fitness have driven maintenance of genetic variation in the selection lines while extending their longevity.

The association between stress resistance and lifespan has motivated the hypothesis that reactive oxygen species (ROS) cause aging [55] and led to experimental tests for lifespan extension by targeting activity of genes that promote antioxidant defenses. For example, overexpression of Catalase (Cat), Superoxide dismutase (SOD), $m s r A$, and glucose-6-phosphate dehydrogenase (G6PD) all increased lifespan in Drosophila [56-59]. Even though we have not found significant polymorphism in these genes, genes with oxidoreductase activities that reduce or block oxygen in different forms from generating free-radical damage belonged to the most overrepresented group among those under the strongest balancing selection. Interestingly, many lncRNAs showed a polymorphism pattern consistent with signatures of balancing selection. Although their functions in Drosophila still await characterization, lncRNAs in mammals play important roles in a wide range of biological processes, including age-related diseases like cancer, cardiovascular pathologies, and neurodegenerative disorders [60]. In cucumber, some lncRNAs seem to be affected by balancing selection as well [61]. 
Drift and balancing selection are by no means the only evolutionary processes capable of increasing the levels of genetic diversity. Positive frequency-dependent selection, as selection for common alleles, is typically predicted to result in monomorphism, and therefore perceived as a process opposite to balancing selection. However, it occasionally may maintain rather than eliminate polymorphism, under the influence of their interactions with other alleles in the system [17]. One could argue that this mechanism may be sufficient to create hitchhiking effects on variability across the genome, especially when LD is increased due to bottlenecking of the experimental populations. Our simulations of this scenario under various LD (recombination) conditions and selection pressures (see Materials and Methods) failed to increase heterozygosity in the in silico longevity lines.

Genetic purging whereby allelic diversity is eroded by negative selection under inbreeding [62] potentially provides another alternative explanation of our results, assuming that purging of early-acting mildly deleterious variants in the control lines was more effective. However, the patterns of heterozygosity and Tajima's D within recombination-suppressed regions are not consistent with purging effects. Since linkage slows down the decline of genetic diversity due to purging [18], one would expect the regions with suppressed recombination to produce the lowest difference in heterozygosity/diversity between longevity and control lines, a pattern opposite to actually observed. For example, the neighborhoods of centromeres representing regions of low recombination on the other chromosomes showed major increases in heterozygosity and Tajima's D in the longevity lines relative to control lines. In another region characterized by a very low level of recombination, the $\sim 71 \mathrm{~Kb}$ yellow-achaete-scute complex near the tip of chromosome X [63], the heterozygosity increase in the longevity lines was even more dramatic $(2,281 \%$ compared with a $47 \%$ increase along the rest of the chromosome X). Detection of balanced polymorphism is enhanced by lower levels of recombination facilitating the correlation between genealogical histories of adjacent SNPs.

The Drosophila fourth chromosome is usually the smallest autosome $(\sim 5 \mathrm{Mb})$, with only a $\sim 1 \mathrm{Mb}$ euchromatic-like region of the right arm containing $\sim 80$ genes, believed to experience no-or very low-rates of recombination [64-68]. As predicted, chromosome 4 was most affected by selection for longevity and showed patters of increased heterozygosity in the selection lines. Notably, another study reported that the genetic variance in viability of $D$. melanogaster for the chromosome 4 was approximately one-half of that for the second chromosome [14], despite the fact that it contains less than $1 / 20$ th the number of genes. The chromosome is variable in several regions forming domains within
$20-30 \%$ of the euchromatic arm with highly dimorphic haplotypes, already presumed to be maintained by balancing selection $[66,68]$.

Despite the paucity of sites with SNP polymorphism patterns that would suggest positive selection in the longevity lines, several genes still seem to have diverged between the selection and control groups through positive selection. The two most prominent were Ptp10D and Ank2 with important roles in central nervous system and memory [36-39, 41].

\section{Conclusions}

In sum, these results show that directional selection for extended life span in D. melanogaster leads to genetic diversification consistent with the operation of balancing selection either through antagonistic pleiotropy or cycling conditions between late reproduction and early reproduction generations. Such balancing selection effects may be prevalent in other 'evolve-and-resequence' experiments in which life history traits are under directional selection, but may go unnoticed when detection of genetic divergence and signatures of positive selection is the main focus of genomic analyses. Processes other than balancing selection, such as drift, genetic purging, and hitchhiking around sites under positive frequency-dependent selection (or their combination) may also be factors contributing to inflated genetic diversity, even if our simulations did not support such a conclusion in the presented case.

\section{Methods}

\section{Drosophila culturing and experimental evolution}

The lines were derived from a mass population of $D$. melanogaster established in our laboratory in September 2002. To ensure ample genetic diversity of the starting material, this population was founded by mixing four pre-existing laboratory stocks (600-700 flies from each). The stocks were discrete or mixed populations from four natural populations, two located in Denmark, one from Australia and one from the Netherlands, maintained in the laboratory in large numbers of breeding individuals. Flies were reared under low to moderately high density on standard Drosophila medium at $25{ }^{\circ} \mathrm{C}$ unless otherwise stated. The four stocks were:

\section{1) Hov-Copenhagen basic strain}

The flies were collected in two sites in Denmark (October 1997), Hvidovre (Zealand island, near Copenhagen) and Hov (Jutland peninsula, east coast). They were kept as 30 and 27 isofemale lines, respectively. The lines were mixed in February 1998 and maintained as one large interbreeding population. 
2) Supermass Hov-Copenhagen population

This population was founded in September 2001 by mixing a number of heat-resistant and longevity selection lines. The heat-resistant selection lines were founded using offspring from the 16th generation of the Hov-Copenhagen basic strain. There were four sets of lines: lines selected for increased survival after heat shock $\left(38.6{ }^{\circ} \mathrm{C}\right)$ with and without prior heat-hardening $\left(37.0{ }^{\circ} \mathrm{C}\right)$, lines that were heat-hardened, but not selected, and lines reared at cycling temperatures (25 and $35{ }^{\circ} \mathrm{C}$ for 18 and $6 \mathrm{~h}$, respectively). From May 2001, the first three sets were maintained without selection and/ or hardening treatment. The longevity selection lines were established in April 2000 by sampling flies from the Hov- Copenhagen basic strain. They were selected for increased lifespan at two temperature regimes, 25 and $29^{\circ} \mathrm{C}$.

\section{3) Heat-knockdown selection lines}

These lines originate from two sets of highly inbred laboratory lines described by Norry et al. [69]. The first of them $(\mathrm{SH})$ was founded by flies collected near Melbourne, Australia in February 1994 and selected for increased heat-knockdown resistance. The second set of lines (D) was founded by flies from the 10th generation of the Hov-Copenhagen basic strain and later selected for reduced heat-knockdown resistance.

\section{4) Leiden strain}

This strain was represented by 30 isofemale lines originating from females collected near Leiden (the Netherlands) in October 1999. For the first five generations it was maintained at $25{ }^{\circ} \mathrm{C}$ and then at $20{ }^{\circ} \mathrm{C}$.

The mass population was maintained at $25{ }^{\circ} \mathrm{C}$ on a standard oatmeal-sugar-yeast-agar Drosophila medium. Every subsequent generation was founded using a mix of parents from different bottles. There were 25 bottles in total with ca. 50 pairs of parents per bottle. The six experimental lines described below (three selection and three control lines) were established by flies from the fourth generation of the mass population.

\section{Selection and control lines}

Each replicate line was maintained in five culture bottles with a minimum population size of 60 pairs in each (in total a population size of 300 pairs). The five bottles, within a replicate line, were mixed each generation. The longevity selection took place every other generation. New emerged flies were placed in food vials and transferred to new vials every second day until approximately
$50 \%$ mortality was reached. In the first generation of selection, this took 4 weeks, and after 48 generations of selection $50 \%$ mortality was reached after approximately 7-8 weeks. The surviving flies were used to start the next generation. Replicate lines of the control regime were allowed to breed within a week from eclosing and kept under standard laboratory conditions at $25^{\circ} \mathrm{C}$ and a 12/12 h light/dark cycle on standard agar-sugar-yeastoatmeal medium. When flies for this experiment were sampled the lines selected for increased longevity had on average a $66 \%$ longer median lifespan than control lines in males and $63 \%$ in females [70, 71]. Flies from the selection group sampled for sequencing were offspring from an unselected generation, after 48 generations of selection (a total of 107 generations). Flies from the control regime used for sequencing were sampled after 201 generations. From each line 500 individuals of equal sex ratio were sampled. The DNA was extracted using the CTAB method in batches of 25 flies and pooled by line. TruSeq DNA libraries were prepared and sequenced on the HiSeq 2000 platform following Illumina's protocols, and 2x90 bp paired-end reads were generated (Additional file 1: Table S2).

\section{Mapping and genotyping}

The Drosophila melanogaster genome (dm3) and corresponding annotations (RefSeq) from UCSC (http://genome. ucsc.edu/) were used as reference for mapping. Raw reads were quality-controlled and filtered with FastqMcf [72]. The remaining reads were mapped to the reference using BWA [73] using default parameters. GATK [74] with default parameters (except for using '-sample_ploidy' for pooled data and setting -heterozygosity to 0.01) was employed to generate genotypes in each line. Genotypes with more than 2 alleles were discarded. Only sites with genotyping quality greater than 30 , minimum depth 10 , and maximum depth 250 were used in the analysis.

\section{Estimates of Fst, $\pi, \theta$, Tajima's D, and heterozygosity}

Samtools [75] was used to generate the pileup result. SNPs within $10 \mathrm{bp}$ of indels were discarded. $\mathrm{F}_{\mathrm{ST}}$ value for each SNP was generated using Poopolation2 [76], while PoPoolation [77] was used to calculate $\pi$, Watterson's $\theta$ and Tajima's D with the window size set to $10 \mathrm{~Kb}$. Heterozygosity was estimated using a $100 \mathrm{~Kb}$ sliding window with a step of $10 \mathrm{~Kb}$.

\section{Sweep region detection}

Putative selective sweep regions were detected with Poolhmm [78], a hidden Markov model for finding selective sweep signatures from Pool-Seq data. The parameters used in Pool-hmm were "-n 100 -c 5 -C 400 -q 20 -e sanger -p -k 0.0000000001", while "-theta" was set to be the $\theta$ estimated for each sample. 


\section{Neutrality and positive frequency-dependent selection simulations}

Genome simulations under a neutrality model were conducted using forqs [79]. The haplotype data for the simulation were obtained from the Drosophila Genetic Reference Panel 2 (DGRP2, http://dgrp2.gnets.ncsu.edu/) of 205 inbreed lines. The mass-breeding phase of the experiment was simulated for 1,000 generations with a population size of 100,000 . Conditions corresponding to our experimental system were simulated for 201 generations with population size limited to 300 individuals. Two types of simulations were performed: 1) To test whether observed patterns of genomic differentiation could be produced by drift alone, the recombination rate was set to 2 and no selection was added. Simulations were run three times to mimic the replicas in our study. A total of 6 chromosomes/arms (chr2L, chr2R, chr3L, chr3R, chr4 and $\mathrm{chrX)}$ were generated. Missing genotypes were set as heterozygous. Heterozygosity and Tajima's D were computed based on the same window size as for the real experimental data and compared between generations 107 and 201, corresponding to the experimental gap between longevity and control lines, respectively. 2) To test the hitchhiking effects of positive frequency-dependent selection in conjunction with increased LD, the recombination rate was set to either 2 or 0.2 for testing under conditions of moderate and low recombination. A $1.1 \mathrm{M}$-size region from chr2L:9,500,000-11,500,000 was simulated, while a total of 22 selected sites were evenly distributed along the region. The initial deleterious allele frequencies for all selected sites were set to 0.1 (under the Hardy-Weinberg equilibrium) and fitness for the deleterious allele was set to 0.9 or 0.999 for mimicking strong selection or weak/no selection. A total of 1,000 generations were simulated and the simulations were repeated 100 times. Heterozygosity was calculated with the same window size $(100 \mathrm{~Kb})$ along this region every 100 generations.

\section{Additional files}

Additional file 1: Table S1. Summary of genes with the largest differential Tajima's D values. Table S2. Summary of analyzed reads. (XLSX $32 \mathrm{~kb}$ )

Additional file 2: Figure S1. Simulations of heterozygosity decrease under neutral evolution. (PNG $152 \mathrm{~kb}$ )

Additional file 3: Figure S2. Simulations of positive frequencydependent selection pressures (strong or weak) under moderate (A) or low (B) recombination rates. (TIFF $5741 \mathrm{~kb}$ )

\section{Acknowledgements}

We are grateful to Doth Andersen for setting up and maintaining the selection and corresponding control lines and to the Danish Natural Sciences Research council and the Carlsberg Foundation for financial support to VL. Sequencing was done by $B G I$.

\section{Funding}

The Danish Natural Sciences Research council and the Carlsberg Foundation for financial support to VL.

\section{Availability of data and materials}

The sequence data supporting the results of this article are available in the NCBI' s Sequence Read Archive (SRA) under the accession number SRP092765.

\section{Authors' contributions}

PM wrote the paper and interpreted the data; LK analyzed the sequence data and contributed to writing; PMS conducted the experiment and contributed to data interpretation and writing; MFS contributed to data interpretation and writing; VL conceived the experiment and contributed to data interpretation and writing. All authors read and approved the final manuscript.

\section{Competing interests}

The authors declare that they have no competing interests.

\section{Consent for publication}

Not applicable.

Ethics approval and consent to participate Not applicable.

Received: 17 September 2016 Accepted: 10 January 2017

Published online: 14 January 2017

\section{References}

1. Rose MR. Evolutionary biology of aging. New York: Oxford University Press; 1991.

2. Charlesworth B. Evolution in age-structured populations. 2nd ed. Cambridge England; New York: Cambridge University Press; 1994.

3. Medawar PB. An unsolved problem of biology. London: Published for the college by H. K. Lewis; 1952.

4. Hamilton WD. The moulding of senescence by natural selection. J Theor Biol. 1966;12(1):12-45.

5. Williams GC. Pleiotropy, Natural-Selection, and the Evolution of Senescence. Evolution. 1957;11(4):398-411.

6. Rose MR. Life-History Evolution with Antagonistic Pleiotropy and Overlapping Generations. Theor Popul Biol. 1985;28(3):342-58.

7. Curtsinger JW, Service PM, Prout T. Antagonistic Pleiotropy, Reversal of Dominance, and Genetic Polymorphism. Am Nat. 1994;144(2):210-28.

8. Hedrick PW. Antagonistic pleiotropy and genetic polymorphism: a perspective. Heredity. 1999;82(2):126-33.

9. Connallon T, Clark AG. Antagonistic versus non-antagonistic models of balancing selection: Characterizing the relative timescales and hitchhiking effects of partial selective sweeps. Evolution. 2013;67(3):908-17.

10. Kelly JK. An experimental method for evaluating the contribution of deleterious mutations to quantitative trait variation. Genet Res. 1999;73(03):263-73.

11. Charlesworth B, Miyo T, Borthwick H. Selection responses of means and inbreeding depression for female fecundity in Drosophila melanogaster suggest contributions from intermediate-frequency alleles to quantitative trait variation. Genet Res. 2007:89(2):85-91.

12. Kelly JK, Willis $\mathrm{JH}$. Deleterious mutations and genetic variation for flower size in Mimulus guttatus. Evolution. 2001;55(5):937-42.

13. Kelly JK. Deleterious mutations and the genetic variance of male fitness components in Mimulus guttatus. Genetics. 2003;164(3):1071-85.

14. Kenyon A. Comparison of frequency distributions of viabilities of second with fourth chromosomes from caged Drosophila melanogaster. Genetics. 1967;55(1):123-30.

15. Charlesworth B. Causes of natural variation in fitness: evidence from studies of Drosophila populations. Proc Natl Acad Sci U S A. 2015;112(6):1662-9.

16. Bergland AO, Behrman EL, O'Brien KR, Schmidt PS, Petrov DA. Genomic evidence of rapid and stable adaptive oscillations over seasonal time scales in Drosophila. PLoS Genet. 2014;10(11), e1004775.

17. Trotter MV, Spencer HG. Frequency-Dependent Selection and the Maintenance of Genetic Variation: Exploring the Parameter Space of the Multiallelic Pairwise Interaction Model. Genetics. 2007;176(3):1729-40.

18. Bersabe D, Caballero A, Perez-Figueroa A, Garcia-Dorado A. On the Consequences of Purging and Linkage on Fitness and Genetic Diversity. G3 (Bethesda). 2016;6(1):171-81. 
19. Bubliy OA, Loeschcke V. Correlated responses to selection for stress resistance and longevity in a laboratory population of Drosophila melanogaster. J Evol Biol. 2005;18(4):789-803.

20. Wit J, Sarup P, Lupsa N, Malte H, Frydenberg J, Loeschcke V. Longevity for free? Increased reproduction with limited trade-offs in Drosophila melanogaster selected for increased life span. Exp Gerontol. 2013;48(3):349-57.

21. Wit J, Kristensen TN, Sarup P, Frydenberg J, Loeschcke V. Laboratory selection for increased longevity in Drosophila melanogaster reduces field performance. Exp Gerontol. 2013;48(11):1189-95.

22. Rose MR. Genetics of Increased Lifespan in Drosophila. Bioessays. 1989;11(5):132-5

23. Paaby $A B$, Schmidt PS. Dissecting the genetics of longevity in Drosophila melanogaster. Fly. 2009;3(1):29-38.

24. Kirkwood TB, Austad SN. Why do we age? Nature. 2000;408(6809):233-8.

25. Partridge L, Gems D. Mechanisms of ageing: Public or private? Nat Rev Genet. 2002;3(3):165-75.

26. Helfand SL, Rogina B. Genetics of aging in the fruit fly, Drosophila melanogaster. Annu Rev Genet. 2003;37:329-48.

27. Burke MK, Dunham JP, Shahrestani P, Thornton KR, Rose MR, Long AD Genome-wide analysis of a long-term evolution experiment with Drosophila. Nature. 2010;467(7315):587-90.

28. Schlotterer C, Kofler R, Versace E, Tobler R, Franssen SU. Combining experimental evolution with next-generation sequencing: a powerful tool to study adaptation from standing genetic variation. Heredity (Edinb). 2015;114(5):431-40.

29. Turner TL, Stewart AD, Fields AT, Rice WR, Tarone AM. Population-based resequencing of experimentally evolved populations reveals the genetic basis of body size variation in Drosophila melanogaster. PLoS Genet. 2011;7(3), e1001336.

30. Remolina SC, Chang PL, Leips J, Nuzhdin SV, Hughes KA. Genomic basis of aging and life-history evolution in Drosophila melanogaster. Evolution. 2012;66(11):3390-403.

31. Jha AR, Miles CM, Lippert NR, Brown CD, White KP, Kreitman M. WholeGenome Resequencing of Experimental Populations Reveals Polygenic Basis of Egg-Size Variation in Drosophila melanogaster. Mol Biol Evol. 2015;32(10):2616-32.

32. Carnes MU, Campbell T, Huang W, Butler DG, Carbone MA, Duncan LH, Harbajan SV, King EM, Peterson KR, Weitzel A, et al. The Genomic Basis of Postponed Senescence in Drosophila melanogaster. PLoS One. 2015;10(9), e0138569.

33. Harshman LG, Hoffmann AA. Laboratory selection experiments using Drosophila: what do they really tell us? Trends Ecol Evol. 2000;15(1):32-6.

34. Houle D. Genetic Covariance of Fitness Correlates: What Genetic Correlations are Made of and Why it Matters. Evolution. 1991;45(3):630-48.

35. Findlay GD, Sitnik JL, Wang W, Aquadro CF, Clark NL, Wolfner MF. Evolutionary Rate Covariation Identifies New Members of a Protein Network Required for Drosophila melanogaster Female Post-Mating Responses. PLoS Genetics. 2014;10(1), e1004108.

36. Jeon M, Nguyen H, Bahri S, Zinn K. Redundancy and compensation in axon guidance: genetic analysis of the Drosophila Ptp10D/Ptp4E receptor tyrosine phosphatase subfamily. Neural Dev. 2008;3:3.

37. Lee HK, Cording A, Vielmetter J, Zinn K. Interactions between a Receptor Tyrosine Phosphatase and a Cell Surface Ligand Regulate Axon Guidance and Glial-Neuronal Communication. Neuron. 2013;78(5):813-26.

38. Qian M, Pan G, Sun L, Feng C, Xie Z, Tully T, Zhong Y. Receptor-like tyrosine phosphatase PTP10D is required for long-term memory in Drosophila. J Neurosci. 2007;27(16):4396-402

39. Yamamoto M, Ueda R, Takahashi K, Saigo K, Uemura T. Control of axonal sprouting and dendrite branching by the Nrg-Ank complex at the neuron-glia interface. Curr Biol. 2006;16(16):1678-83.

40. Neely GG, Hess A, Costigan M, Keene AC, Goulas S, Langeslag M, Griffin RS, Belfer I, Dai F, Smith SB, et al. A Genome-wide Drosophila Screen for Heat Nociception Identifies ?2?3 as an Evolutionarily Conserved Pain Gene. Cell. 2010;143(4):628-38.

41. Iqbal Z, Vandeweyer G, van der Voet M, Waryah AM, Zahoor MY, Besseling JA, Roca LT, Vulto-van Silfhout AT, Nijhof B, Kramer JM, et al. Homozygous and heterozygous disruptions of ANK3: at the crossroads of neurodevelopmental and psychiatric disorders. Hum Mol Genet. 2013;22(10):1960-70.

42. Kang L, Aggarwal DD, Rashkovetsky E, Korol AB, Michalak P. Rapid genomic changes in Drosophila melanogaster adapting to desiccation stress in an experimental evolution system. BMC Genomics. 2016;17(1):233.
43. Asthana S, Schmidt S, Sunyaev S. A limited role for balancing selection. Trends Genet. 2005:21(1):30-2.

44. Andres AM, Hubisz MJ, Indap A, Torgerson DG, Degenhardt JD, Boyko AR, Gutenkunst RN, White TJ, Green ED, Bustamante CD, et al. Targets of balancing selection in the human genome. Mol Biol Evol. 2009;26(12):2755-64.

45. Roux C, Pauwels M, Ruggiero MV, Charlesworth D, Castric V, Vekemans X. Recent and ancient signature of balancing selection around the S-locus in Arabidopsis halleri and A. lyrata. Mol Biol Evol. 2013;30(2):435-47.

46. Akey JM, Zhang G, Zhang K, Jin L, Shriver MD. Interrogating a highdensity SNP map for signatures of natural selection. Genome Res. 2002;12(12):1805-14

47. Paaby $A B$, Schmidt PS. Dissecting the genetics of longevity in Drosophila melanogaster. Fly (Austin). 2009;3(1):29-38.

48. Roff DA. The evolution of life histories: theory and analysis. New York: Chapman \& Hall; 1992

49. Stearns SC. The evolution of life histories. Oxford: Oxford University Press; 1992.

50. Reznick D. Costs of Reproduction - an Evaluation of the Empirical-Evidence. Oikos. 1985;44(2):257-67.

51. Loeschcke V. Genetic constraints on adaptive evolution. Berlin: Springer; 1987.

52. Stearns SC. Trade-Offs in Life-History Evolution. Funct Ecol. 1989;3(3):259-68.

53. Vermeulen CJ, Loeschcke V. Longevity and the stress response in Drosophila. Exp Gerontol. 2007:42(3):153-9.

54. Tobler R, Franssen SU, Kofler R, Orozco-Terwengel P, Nolte V, Hermisson J, Schlotterer C. Massive habitat-specific genomic response in D. melanogaster populations during experimental evolution in hot and cold environments. Mol Biol Evol. 2014;31(2):364-75.

55. Harman D. The aging process. Proc Natl Acad Sci U S A. 1981;78(11):7124-8.

56. Orr WC, Sohal RS. Extension of life-span by overexpression of superoxide dismutase and catalase in Drosophila melanogaster. Science. 1994; 263(5150):1128-30.

57. Parkes TL, Elia AJ, Dickinson D, Hilliker AJ, Phillips JP, Boulianne GL. Extension of Drosophila lifespan by overexpression of human SOD1 in motorneurons. Nat Genet. 1998;19(2):171-4.

58. >Ruan $\mathrm{H}$, Tang XD, Chen ML, Joiner ML, Sun G, Brot N, Weissbach $H_{\text {, }}$ Heinemann SH, Iverson L, Wu CF, et al. High-quality life extension by the enzyme peptide methionine sulfoxide reductase. Proc Natl Acad Sci U S A. 2002;99(5):2748-53.

59. Legan SK, Rebrin I, Mockett RJ, Radyuk SN, Klichko VI, Sohal RS, Orr WC. Overexpression of glucose-6-phosphate dehydrogenase extends the life span of Drosophila melanogaster. J Biol Chem. 2008;283(47):32492-9.

60. Grammatikakis I, Panda AC, Abdelmohsen K, Gorospe M. Long noncoding RNAs(IncRNAs) and the molecular hallmarks of aging. Aging (Albany NY). 2014;6(12):992-1009.

61. Hao Z, Fan C, Cheng T, Su Y, Wei Q, Li G. Genome-wide identification, characterization and evolutionary analysis of long intergenic noncoding RNAs in cucumber. PLoS One. 2015;10(3), e0121800.

62. Crnokrak P, Barrett SC. Perspective: purging the genetic load: a review of the experimental evidence. Evolution. 2002;56(12):2347-58.

63. Macpherson JN, Weir BS, Leigh Brown AJ. Extensive linkage disequilibrium in the achaete-scute complex of Drosophila melanogaster. Genetics. 1990;126(1):121-9.

64. Berry AJ, Ajioka JW, Kreitman M. Lack of polymorphism on the Drosophila fourth chromosome resulting from selection. Genetics. 1991;129(4):1111-7.

65. Jensen MA, Charlesworth B, Kreitman M. Patterns of genetic variation at a chromosome 4 locus of Drosophila melanogaster and D. simulans. Genetics. 2002;160(2):493-507.

66. Wang W, Thornton K, Berry A, Long M. Nucleotide Variation Along the Drosophila melanogaster Fourth Chromosome. Science. 2002; 295(5552):134-7.

67. Haddrill PR, Halligan DL, Tomaras D, Charlesworth B. Reduced efficacy of selection in regions of the Drosophila genome that lack crossing over. Genome Biol. 2007;8(2):R18.

68. Arguello JR, Zhang Y, Kado T, Fan C, Zhao R, Innan H, Wang W, Long M. Recombination yet inefficient selection along the Drosophila melanogaster subgroup's fourth chromosome. Mol Biol Evol. 2010;27(4):848-61.

69. Norry FM, Dahlgaard J, Loeschcke V. Quantitative trait loci affecting knockdown resistance to high temperature in Drosophila melanogaster. Mol Ecol. 2004;13(11):3585-94.

70. Sarup P, Loeschcke V. Life extension and the position of the hormetic zone depends on sex and genetic background in Drosophila melanogaster. Biogerontology. 2011;12(2):109-17. 
71. Sarup P, Sorensen P, Loeschcke V. Flies selected for longevity retain a young gene expression profile. Age (Dordr). 2011;33(1):69-80.

72. Aronesty E. Comparison of Sequencing Utility Programs. Open Bioinforma J. 2013;7:1-8.

73. Li H, Durbin R. Fast and accurate short read alignment with Burrows-Wheeler transform. Bioinformatics. 2009;25(14):1754-60

74. DePristo MA, Banks E, Poplin R, Garimella KV, Maguire JR, Hartl C, Philippakis AA, del Angel G, Rivas MA, Hanna M, et al. A framework for variation discovery and genotyping using next-generation DNA sequencing data. Nat Genet. 2011;43(5):491-8.

75. Li H, Handsaker B, Wysoker A, Fennell T, Ruan J, Homer N, Marth G, Abecasis G, Durbin R, Genome Project Data Processing S. The Sequence Alignment/Map format and SAMtools. Bioinformatics. 2009;25(16):2078-9.

76. Kofler R, Pandey RV, Schlotterer C. PoPoolation2: identifying differentiation between populations using sequencing of pooled DNA samples (Pool-Seq). Bioinformatics. 2011;27(24):3435-6.

77. Kofler R, Orozco-terWengel P, De Maio N, Pandey RV, Nolte V, Futschik A, Kosiol C, Schlotterer C. PoPoolation: a toolbox for population genetic analysis of next generation sequencing data from pooled individuals. PLoS One. 2011;6(1), e15925.

78. Boitard S, Kofler R, Francoise P, Robelin D, Schlotterer C, Futschik A. Pool-hmm: a Python program for estimating the allele frequency spectrum and detecting selective sweeps from next generation sequencing of pooled samples. Mol Ecol Resour. 2013;13(2):337-40.

79. Kessner D, Novembre J. forqs: forward-in-time simulation of recombination, quantitative traits and selection. Bioinformatics. 2014;30(4):576-7.

\section{Submit your next manuscript to BioMed Central and we will help you at every step:}

- We accept pre-submission inquiries

- Our selector tool helps you to find the most relevant journal

- We provide round the clock customer support

- Convenient online submission

- Thorough peer review

- Inclusion in PubMed and all major indexing services

- Maximum visibility for your research

Submit your manuscript at www.biomedcentral.com/submit

) Biomed Central 